\title{
Genome-scale model-driven strain design for dicarboxylic acid production in Yarrowia lipolytica
}

Pranjul Mishra ${ }^{1 \dagger}$, Na-Rae Lee ${ }^{1 \dagger}$, Meiyappan Lakshmanan², Minsuk Kim³ ${ }^{3}$, Byung-Gee Kim³ and Dong-Yup Lee ${ }^{1,24^{*}}$

From The 28th International Conference on Genome Informatics

Seoul, Korea. 31 October - 3 November 2017

\begin{abstract}
Background: Recently, there have been several attempts to produce long-chain dicarboxylic acids (DCAs) in various microbial hosts. Of these, Yarrowia lipolytica has great potential due to its oleaginous characteristics and unique ability to utilize hydrophobic substrates. However, Y. lipolytica should be further engineered to make it more competitive: the current approaches are mostly intuitive and cumbersome, thus limiting its industrial application.

Results: In this study, we proposed model-guided metabolic engineering strategies for enhanced production of DCAs in $Y$. lipolytica. At the outset, we reconstructed genome-scale metabolic model (GSMM) of Y. lipolytica (YLL1647) by substantially expanding the previous models. Subsequently, the model was validated using three sets of published culture experiment data. It was finally exploited to identify genetic engineering targets for overexpression, knockout, and cofactor modification by applying several in silico strain design methods, which potentially give rise to high yield production of the industrially relevant long-chain DCAs, e.g., dodecanedioic acid (DDDA). The resultant targets include (1) malate dehydrogenase and malic enzyme genes and (2) glutamate dehydrogenase gene, in silico overexpression of which generated additional NADPH required for fatty acid synthesis, leading to the increased DDDA fluxes by $48 \%$ and $22 \%$ higher, respectively, compared to wild-type. We further investigated the effect of supplying branched-chain amino acids on the acetyl-CoA turn-over rate which is key metabolite for fatty acid synthesis, suggesting their significance for production of DDDA in Y. lipolytica.

Conclusion: In silico model-based strain design strategies allowed us to identify several metabolic engineering targets for overproducing DCAs in lipid accumulating yeast, Y. lipolytica. Thus, the current study can provide a methodological framework that is applicable to other oleaginous yeasts for value-added biochemical production.
\end{abstract}

Keywords: Yarrowia lipolytica, Dicarboxylic acid, Genome-scale metabolic models, Strain design, Metabolic engineering

\footnotetext{
* Correspondence: dongyuplee@skku.edu

${ }^{\dagger}$ Equal contributors

'NUS Synthetic Biology for Clinical and Technological Innovation (SynCTI),

Life Sciences Institute, National University of Singapore, 28 Medical Drive,

Singapore 117456, Singapore

${ }^{2}$ Bioprocessing Technology Institute, Agency for Science, Technology and

Research (A*STAR), 20 Biopolis Way, \#06-01, Centros, Singapore 138668,

Singapore

Full list of author information is available at the end of the article
} 


\section{Background}

Long-chain dicarboxylic acids (DCAs) are widely used in the manufacturing of polyamides and polyesters as the monomeric intermediates [1]. The most commonly employed chemical process to produce DCAs is the ring-opening oxidation of cyclic compounds. However, it requires expensive starting material as well as the environmentally hazardous procedures [2]. Alternatively, DCAs can be synthesized through bio-routes in various microbial hosts, such as Candida sp. [3, 4], Yarrowia lipolytica [5], Pseudomonas aeruginosa [6], Cyptococcus neoformans [7], and Escherichia coli [8]. Of these, recently, $Y$. lipolytica has attracted great attention as a cell factory to manufacture DCAs [9] since this oleaginous yeast is capable of accumulating large amounts of lipids and possess a unique $\omega$-oxidation pathway to catalyze the hydrophobic substrates such as n-alkane and fatty acids [10-13]. In $\omega$-oxidation pathway, after the oxidation of $\omega$-terminal of the fatty acid, fatty acid aldehyde is produced from $\omega$-hydroxy fatty acid by fatty alcohol oxidase, followed by its oxidization to DCAs by NADdependent fatty aldehyde dehydrogenase [14].

Indeed, $Y$. lipolytica as oleaginous yeast has huge potential to become a model organism to produce fattyacid derived products including DCAs. In order to make it industrially competitive, its productivity should be further enhanced, which can be achieved by modification of relevant target genes. However, metabolic engineering of $Y$. lipolytica has been mainly focused on over-producing lipids, e.g., triacylglycerols (TAGs) with only a handful of studies for the increased DCAs production [15]. In addition, most of genetic engineering targets have been identified in an intuitive or ad hoc manner, which limited our design scope for strain improvement. Therefore, it is now imperative to adopt more rational systems approaches [16]. In this regard, various strain design strategies guided by in silico genome-scale metabolic model (GSMM) have been developed and successfully applied to several industrial hosts including $E$. coli $[17,18]$ and S. cerevisiae $[19,20]$. Similarly, in this work, we exploited such in silico methods using GSMM of $Y$. lipolytica which was newly reconstructed by substantially expanding the previous models, thus allowing us to identify various genetic engineering targets for overexpression, knockout, and cofactor modification towards DCA overproduction.

\section{Results and discussion}

\section{Genome-scale metabolic reconstruction of $Y$. lipolytica}

We have reconstructed a genome-scale metabolic model of $Y$. lipolytica for designing DCA overproducing strains. Initially, four existing $Y$. lipolytica GSMMs ( $i$ NL895 [21], $i$ YL619 [22], iMK735 [23] and iYALI4 [24]) were compared on the basis of their gene annotations (see
Methods and Fig. 1a). While $i$ NL895 was developed from the phylogenetically distant yeast $S$. cerevisiae model, $i$ YL619 was reconstructed based on biochemical information databases such as KEGG and BRENDA. $i$ MK735 was completely derived from $S$. cerevisiae model $i$ ND750 [25] by adding a few reactions related to alkane uptake and lipid metabolism whereas iYALI4 stands a bit different from other three models since it was built automatically using RAVEN toolbox from yeast consensus model as template. After qualitative and quantitative assessment, $i$ MK735 was selected as suitable scaffold model since it has wider coverage of cellular physiology of $Y$. lipolytica with better quality in terms of representation and prediction quality.

With the aim of building an objective-oriented model for simulating DCAs production, we first added 9 biosynthetic reactions which are sequentially catalyzed by hydroxylase, oxidase and dehydrogenase enzymes within the $\omega$-oxidation pathway, thus capturing the oxidation of fatty acids to DCAs. The 27 DCAs degradation reactions were also included to represent $\beta$-oxidation pathway together with 6 dicarboxylic acids transport and an exchange reaction pertaining to DDDA secretion. Based on the scaffold model, Y. lipolytica appeared to not degrade leucine and other BCAA into acetyl-CoA, but there are reports implicating the biosynthetic capacity of leucine as an effector of lipogenic capacity in oleaginous organisms [26]; a putative acetyl-CoA producing leucine degradation pathway was recently identified [27]. Therefore, we have added 4 relevant enzymatic reactions for leucine degradation in the model. In total, we added 50 new reactions to the scaffold model mainly related to $\omega$-oxidation and BCAA degradation.

During the manual curation, we removed 55 reactions which are responsible for dead-ends or futile cycles without literature evidence. For example, lactaldehyde dehydrogenase was deleted since there is not enough experimental evidence to support the existence of such reaction in $Y$. lipolytica. Additionally, we corrected the elemental balance and directionality of 45 reactions. For example, the directionality of THRA representing the threonine aldolase mediated breakdown of threonine to glycine and acetaldehyde, was changed to opposite direction favoring acetaldehyde formation based on the direction reported in S. cerevisiae [28]. All the changes made from the scaffold are provided in Additional file 1 and summarized in Fig. 1b. Finally, the resulting in silico $Y$. lipolytica model (iYLI647) consists of 1347 reactions and 1152 metabolites encoded by 647 genes (Fig. 1c). The iYLI647 is available as Systems Biology Markup Language (SBML) file (Additional file 2).

The model predictability of cell growth relies highly on the accuracy of biomass equation. However, we found that the biggest shortcoming of the previous models is 

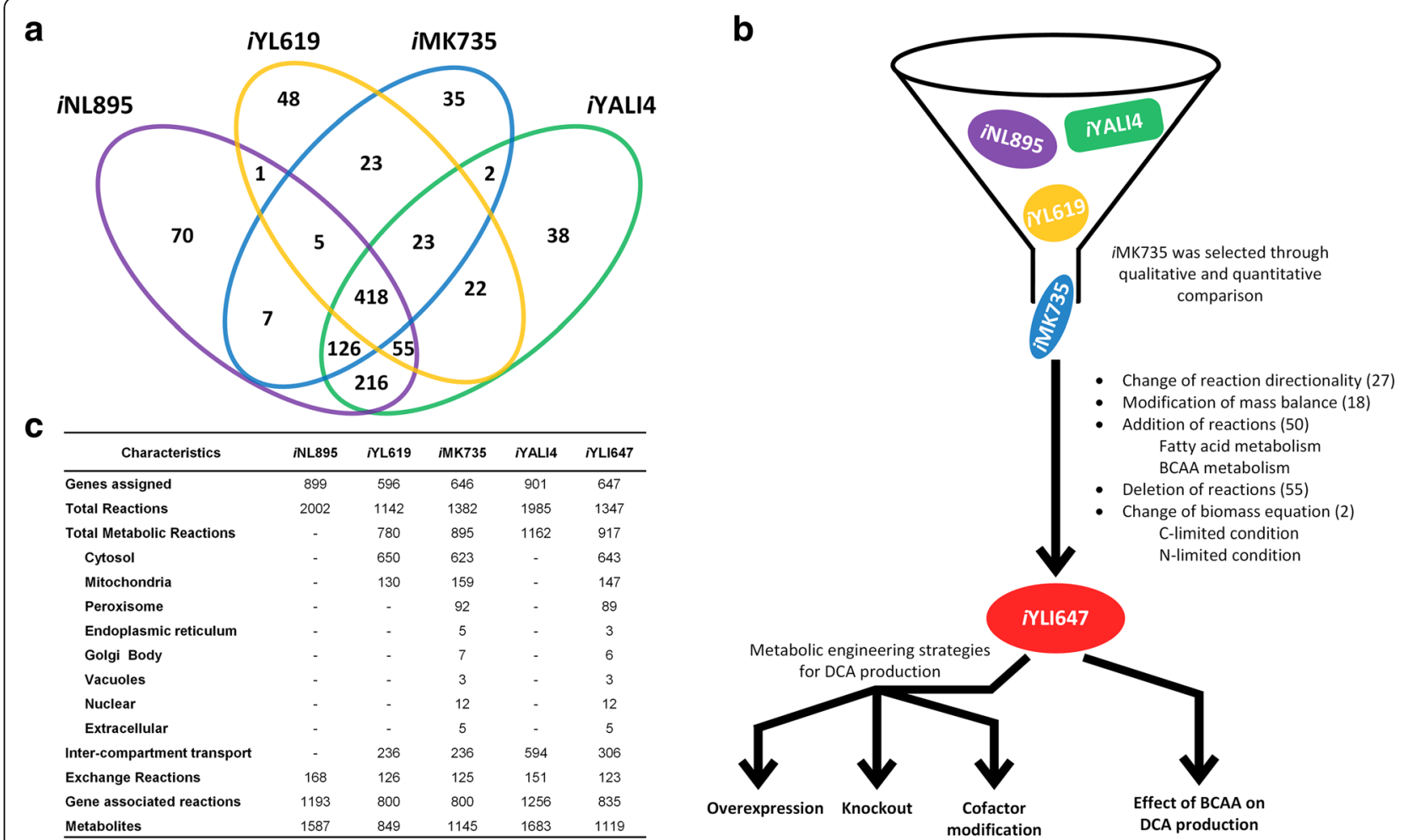

Fig. 1 Reconstruction process and characteristic of in silico models a Comparison of previous Y. lipolytica models b Schematic diagram highlighting the overall reconstruction process of M LL647, followed by application for strain designing c General features of M YLI647 in comparison with previous four models

the inaccurate biomass composition since they mainly used the information of S. cerevisiae biomass. Hence, the whole biomass compositions of $Y$. lipolytica were derived based on various $Y$. lipolytica experimental data published under carbon limited and nitrogen limited conditions which can significantly alter the amino acid and lipid composition. The calculated compositions were then assimilated into iYLI647 as two separate biomass synthesis equations pertaining to C-limited and $\mathrm{N}$ limited conditions (Additional file 3). Growth and nongrowth associated ATP maintenance (GAM and NGAM) requirements for cellular processes were also derived using relevant literature data [29]. The NGAM of $Y$. lipolytica was estimated to be $5.03 \mathrm{mmol} \mathrm{ATP} / \mathrm{gDCW}$, and GAM was $23.09 \mathrm{mmol} \mathrm{ATP} / \mathrm{gDCW}$.

\section{Comparative validation of $i$ YLI647 with Other Y. lipolytica GSMMs}

The iYLI647 was evaluated for its predictions correlated with experimental phenotypes. The growth predictions under different culture conditions of iYLI647 were compared with those for other Y. lipolytica GSMMs. To do so, the in silico biomass yields of all the models on glucose and glycerol minimal medium under steadystate conditions were predicted using FBA. For each set of culture data taken from independent studies, the carbon source uptake rates were constrained accordingly, while maximizing biomass. Additionally, the $\mathrm{CO}_{2}$ evolution rate (CER) was also constrained according to the experimental data wherever provided. For the biomass maximization in $i \mathrm{MK} 735$, biomass equation corresponding to $5 \%$ lipid was used. The maximum specific growth rate was determined for all models simulated under same constraints and the results were compared (Fig. 2).

Datasets 1 and 2 were taken from Workman et al., [30] in which $Y$. lipolytica was grown in batch culture on glycerol and glucose as a sole carbon source, respectively. During the batch culture with glucose, only biomass and $\mathrm{CO}_{2}$ were produced as no metabolite production was observed. In the case of glycerol, apart from biomass and $\mathrm{CO}_{2}$, small amount of polyols in the form of mannitol and arabitol, were also produced. Since, the individual breakdown of polyols was not mentioned in the literature and amounts were small enough to drastically affect the result, we didn't take it into consideration for simulation. Dataset 3 was taken from Dulermo et al., [31] which endeavored to analyze the $Y$. lipolytica mutants for fatty acid production. The carbon source uptake and CER were constrained as per the values reported in the literature and biomass was maximized. 


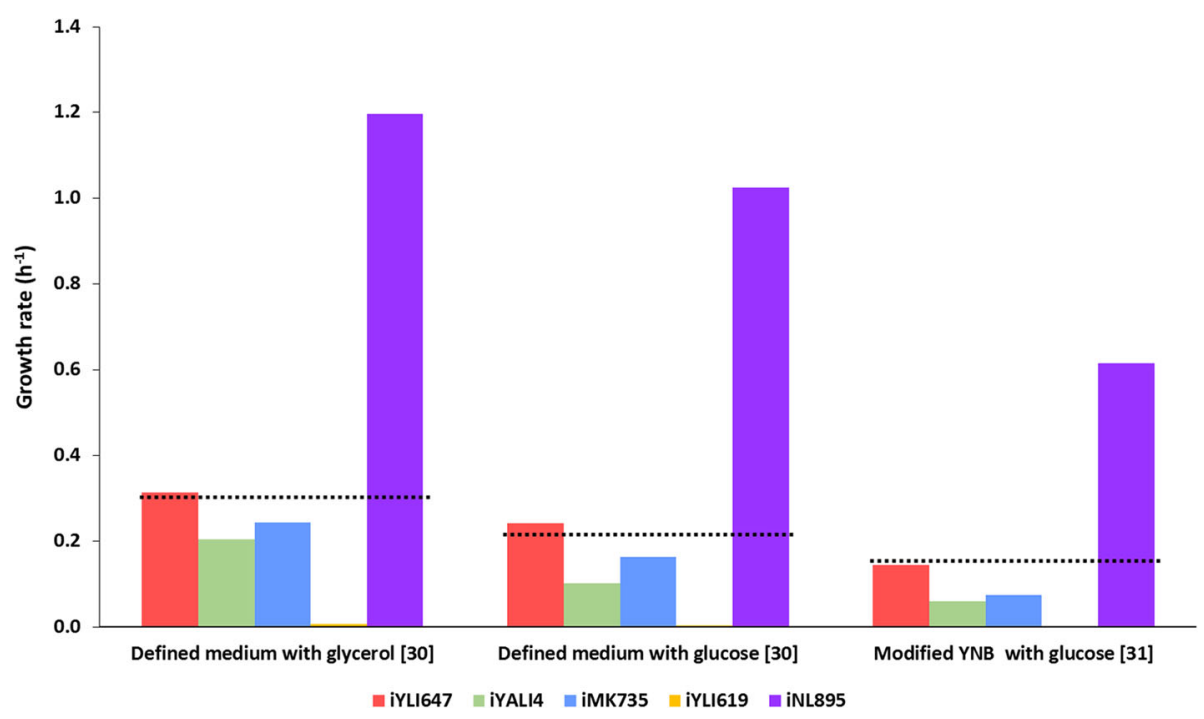

Fig. 2 Comparative validation of MLL647 with all 4 Y. lipolytica models available under 3 different datasets. The dotted lines represent experimental value

It can be seen from comparative validation that $i$ YLI647 can predict the evaluated macroscopic growth parameters more accurately, i.e. smaller deviations from experimental data shown in black dotted lines, as compared to other models. We believe the growth predictions are directly influenced by the accuracy of the biomass composition, and especially, in case of Y. lipolytica the biomass composition can drastically change depending on the culture condition. Another important factor that affects the predictions is the GAM and NGAM values used in the model. Although, in comparative prediction done in the current study, we have used same NGAM in all the models but GAM value was unchanged, and some models reported very high GAM value, resulting in discrepancies in the model prediction.

\section{Metabolic engineering strategies for DCAs production}

In the oleaginous organism, de novo accumulation of lipids starts with the formation of anabolic acetyl-CoA via glycolysis. The fatty acids formed by these acetylCoA get esterified to form TAGs [32]. A high number of carbon sources excluding cellulose and methanol, have been considered as substrates for the de novo DCAs biosynthesis in oleaginous microorganisms. Among the various carbon sources available, we used glucose to design the metabolic engineering strategies for de novo overproduction of DDDA, a representative of long-chain DCAs. Herein, we applied three model-guided design strategies to overproduce DDDA using four tools. First, we employed genetic design by local search (GDLS) [33] to find the growth-coupled solution to overproduce DCAs by knocking out a set of reactions. Then, we used flux activity analysis [34] to identify the bottlenecks in the metabolic network which can be considered as potential overexpression targets. In addition, we implemented transcriptomic-based strain optimization tool (tSOT) [35] which first generates activated reactions as a reference state to identify the deactivated reactions, addition of the each deactivated reactions can then lead to the increase in the product yield. Finally, to supplement the metabolic engineering targets with cofactor availability, we performed cofactor modification analysis (CMA) [36] to find the cofactor specificity engineering targets that can increase the pool of cofactors required for catalyzing the reactions. Figure 3 illustrates the central metabolic network of $Y$. lipolytica and identified genetic engineering targets for enhancing DCAs production.

\section{Overexpression targets}

Expression level of genes and the activity of their enzyme products are highly optimized to meet the performance demand of a biological system [37]. However, these enzyme expressions alter to adapt against the changing biological conditions. This range of fluctuations in gene expression level can be exploited to design an overexpression system [38]. Based on this principle, we performed flux activity analysis under glucose minimal medium by fixing an optimal biomass and systematically increasing flux activity of each reaction from $0 \%$ to $100 \%$ and maximizing DDDA. Simulation results show that when $Y$. lipolytica growing in $10 \mathrm{mmol} /$ gDCW-hr of glucose, the increase in flux activity of some reactions has proportional effect on the maximum achievable yield of DDDA (Fig. 4). These directionally coupled reactions can be genetically overexpressed to complement the $\omega$-oxidation pathway enzymes to produce DDDA at its theoretical maximum. Some of the bottleneck reactions, e.g., DDCAH, a cytochrome P450 


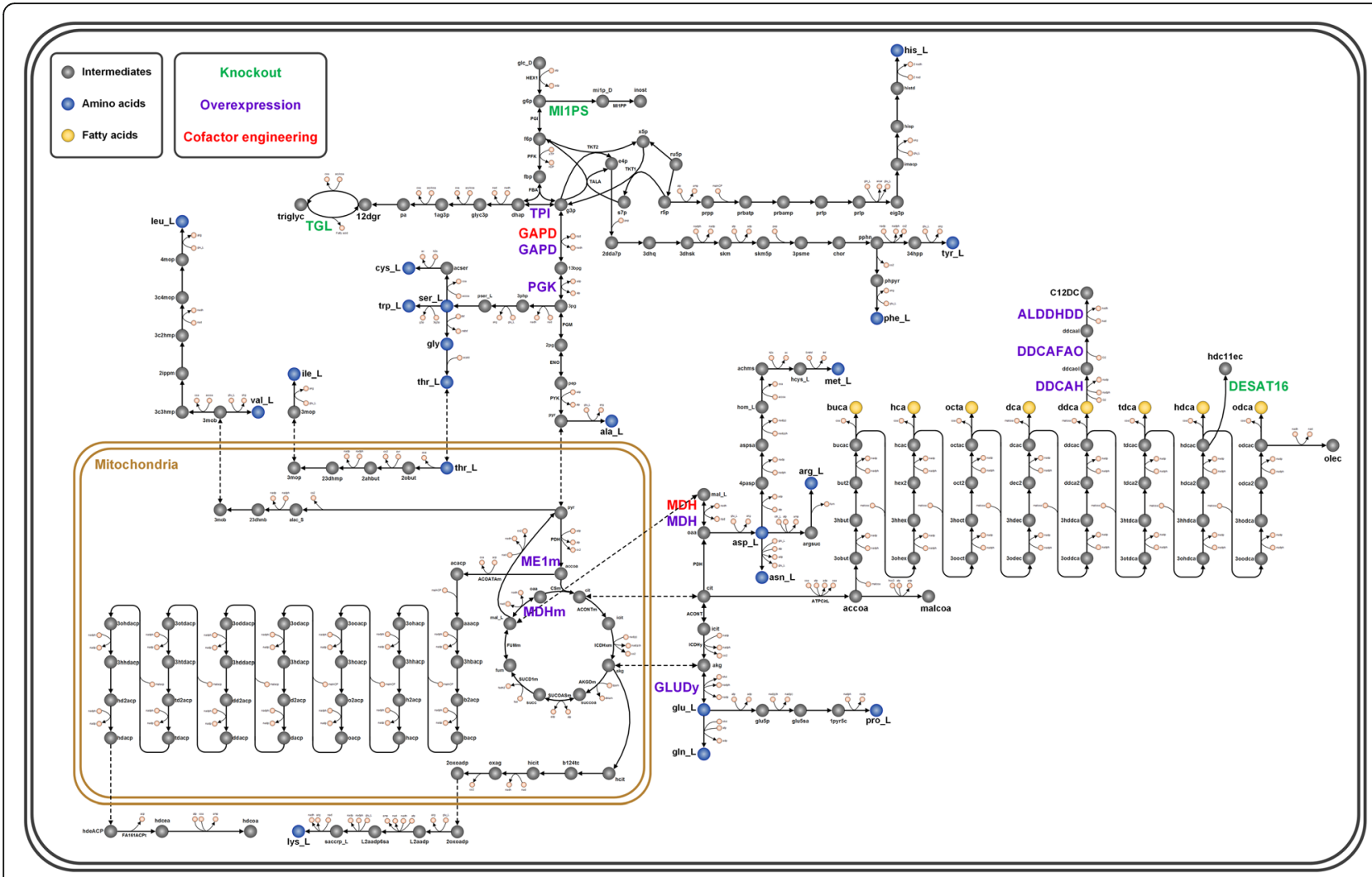

Fig. 3 The central metabolic network of Y. lipolytica depicting metabolic engineering targets to produce DDDA

hydroxylase (CYP52) and DDCAFAO, a fatty alcohol oxidase identified by in silico analysis have been verified as overexpression targets for DDDA production [39]. The other hypothesized reactions include acetyl-CoA carboxylase (ACCOAC), overexpression of which may increase the malonyl-CoA pool. It can be further utilized by FAS complex to generate more fatty acids, which can then be channelized to $\omega$-oxidation pathway.

Considering that fatty acid biosynthetic pathway is very tightly regulated, transcriptomic data can provide some useful insight into the ON/OFF state of the reactions in a particular condition. To identify the overexpression targets

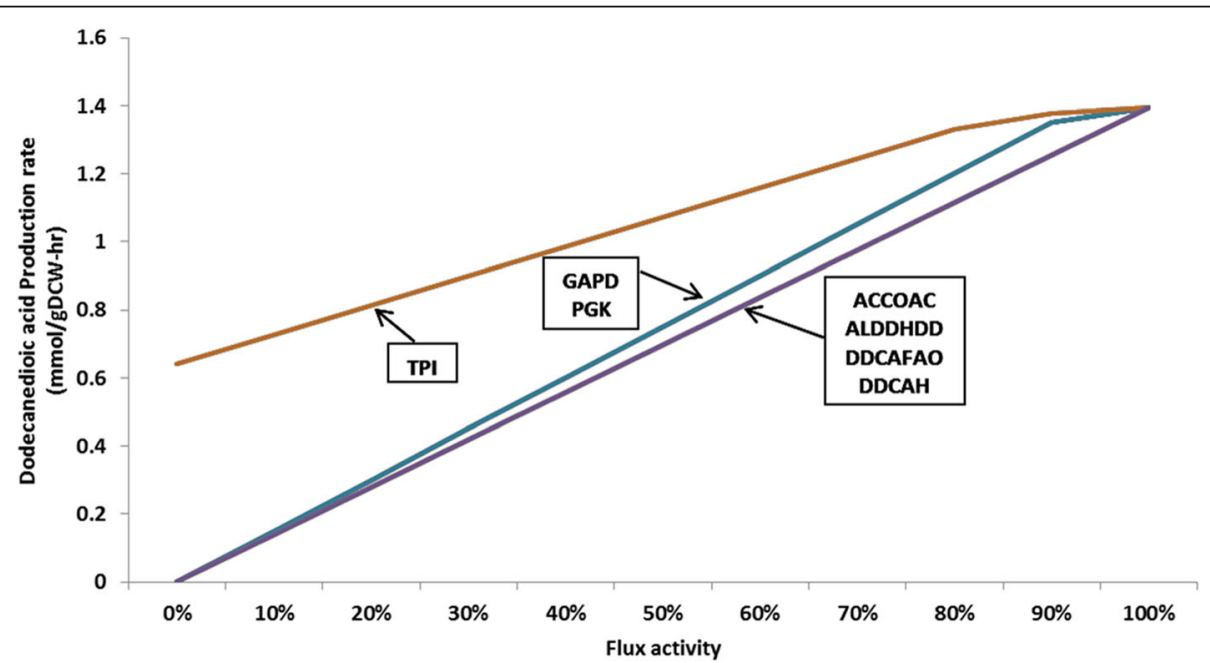

Fig. 4 Simulation result by flux activity analysis. Overexpression genes and production rate changes depend on the alteration of flux activities of respective genes. GAPD (Glyceraldehyde-3-phosphate dehydrogenase), PGK (Phosphoglycerate kinase), TPI (Triose phosphate kinase), ACCOAC (Acetyl-CoA carboxylase), ALDDHDD (Aldehyde dehydrogenase), DDCAFAO (Fatty acid oxidase), DDCAH (Fatty acid hydroxylase) 
for increasing the DCAs production, we implemented tSOT by resorting to time-course transcriptomic profile of the $Y$. lipolytica, during a controlled fed-batch using glucose as the sole carbon source after $27 \mathrm{~h}$ time-point which corresponds to early stationary phase [40]. A nitrogen limitation was applied during the fed-batch to initiate de novo lipid synthesis which can represent the precondition for high DCAs production. The basic principle of tSOT is to ascertain the gene overexpression targets by restoring the reactions which are removed from GSMM by dataintegration algorithms while developing a context-specific model. As a result, tSOT identified MDH, both cytosolic and mitochondrial, as an overexpression target. In addition, it also found mitochondrial NAD-dependent malic enzyme (ME1m) and glutamate dehydrogenase (GLUDy) to be the overexpression targets (Table 1). Interestingly, owing to the fact that $\omega$-oxidation is an oxidative process with the high demand of redox cofactors, all the identified reactions are involved in cofactor regeneration. ME is hypothesized to be the supplier of NADPH during lipid biosynthesis in most oleaginous yeasts through the intracellular substrate cycles involving $\mathrm{MDH}$, pyruvate carboxylase (PC) and ME, also called "transhydrogenase cycle" [41]. Although Y. lipolytica lacks a cytosolic copy of ME required to compensate for $\mathrm{NADPH}$ demand, it could be interesting to investigate the compound effect of overexpressing MDH and ME because apart from NADPH, ME in conjunction with mitochondrial pyruvate dehydrogenase $(\mathrm{PDH})$ also provide mitochondrial acetyl-CoA. The most interesting finding from tSOT is GLUDy, which apart from regeneration of NADPH; also plays key role in maintaining the balance of carbon and nitrogen. There is considerable evidence of the presence of GLUDy shunt in plants, which returns the carbon in amino acids biosynthesis back into reactions of carbon metabolism and TCA cycle [42] which is the case in nitrogen starving condition [24], making it particularly an interesting target to explore as it links amino acid biosynthesis to fatty acid metabolism.

\section{Downregulation/knockout targets}

GDLS algorithm was used to search for growth-coupled solutions for DCA production, identifying up to 5 reaction deletion candidates. Basically, GDLS propose the pathway design which couples the product formation with the cell growth, making its production necessary to reach optimal growth. The strain design strategy deciphered by GDLS combined the simultaneous knockout of DESAT16 (Stearoyl-CoA desaturase) and MI1PS (Myo-inositol-1-phosphate synthase). DESAT16 is the enzyme that catalyzes the conversion of saturated fatty acid to monounsaturated fatty acids. The overexpression of this enzyme is shown to increase lipid accumulation in Y. lipolytica which apparently takes the flux away from DCAs production which requires free fatty acid [43], deleting which can result in increased pool of free fatty acids. Since DCAs is a nongrowth coupled product, deletion of DESAT16 and MI1PS as suggested by GDLS may not give rise to improved product yield. However, in the simple network perspective, DESAT16 and MI1PS reactions branch the carbon flux away from DCAs formation which makes them interesting knockout targets to test experimentally. It is worth noticing here that MI1PS catalyzes the first reaction in the inositol pathway which produces membrane forming metabolites, so knocking out MI1PS could show deleterious effects on cell growth. Nonetheless, since DCAs is non-growth associated product, an inducible knockout strain could be used wherein MI1PS is suppressed when the culture reaches stationary phase.

Furthermore, formed DCAs can get degraded via $\beta$-oxidation pathway. Therefore blocking it by deletion of acyl-CoA oxidase encoded by POX1-6 genes can further enhance the titer of DCAs. Y. lipolytica which lacks acyl-CoA oxidases can more efficiently convert $\mathrm{n}$-alkanes and fatty acids or their derivatives to their corresponding DCAs [5].

\section{Cofactor specificity engineering targets}

Similar to other biosynthetic pathways, the DCAs biosynthesis via $\omega$-oxidation pathway in $Y$. lipolytica involves several unique reactions and is commonly controlled by the supply of precursors and cofactors. Earlier part of this study has focused on overexpression and downregulation of some key enzymes regulating the $\omega$-oxidation pathway. However, cofactors are very important to achieve improvement in productivity. $\mathrm{NADPH}$, as a reducing equivalent, usually plays an important role in coupling catabolism with anabolism and energy generation during metabolism. Several metabolic engineering approaches have been implemented to manipulate the cofactors level to increase the product yield in other microorganisms [44, 45] Increasing the $\alpha$-santalene production by modifying the ammonium

Table 1 Overexpression targets simulated by tSOT to increase DCAs production

\begin{tabular}{|c|c|c|c|}
\hline Targets & Reaction Name & Reaction Definition & Yield Improvement (\%) \\
\hline$\overline{\text { GLUDY }}$ & Glutamate Dehydrogenase (NADPH-forming) & glu_L[c] + h2o[c] + nadp[c] $<=>\operatorname{akg}[c]+h[c]+\operatorname{nadph}[c]+\operatorname{nh} 4[c]$ & 22.2 \\
\hline $\mathrm{MDH}$ & Malate Dehydrogenase (cytosol) & mal_L[c] $+\operatorname{nad}[c]<=>h[c]+\operatorname{nadh}[c]+\operatorname{oaa}[c]$ & 47.8 \\
\hline $\mathrm{MDHm}$ & Malate Dehydrogenase (mitochondrial) & mal_L[m] $+\operatorname{nad}[m]<=>h[m]+\operatorname{nadh}[m]+$ oaa $[m]$ & 47.8 \\
\hline ME1m & Malic enzyme (NAD-dependent) & mal_L $[\mathrm{m}]+\operatorname{nad}[\mathrm{m}]->\operatorname{co} 2[\mathrm{~m}]+\operatorname{nadh}[\mathrm{m}]+\operatorname{pyr}[\mathrm{m}]$ & 47.8 \\
\hline
\end{tabular}


assimilation from being NADPH to NADH dependent by the deletion of GDH1 and the overexpression of GDH2; overexpression of Streptococcus mutants gapN gene which encodes a GAPDH to increase the L-lysine production [46]; engineering NADPH regeneration for improving pentose fermentation by overexpressing the GDP1, a NADP-dependent GAPDH from Kluyveromyces lactis [47]; overexpression of transhydrogenase and NAD kinase to improve isobutanol production [48], are a few examples showing the emergence of NADPH level engineering as potent and feasible strategy to increase the production in microbial hosts.

Fatty acid biosynthesis and $\omega$-oxidation pathway are $\mathrm{NADPH}$ demanding oxidative pathway in $Y$. lipolytica. This excessive cofactor demand is mainly satisfied through the pentose phosphate pathway (PPP) reactions. But, the increased usage of the PPP through overexpression is suboptimal as one mole of carbon is lost as $\mathrm{CO}_{2}$ for every two moles of NADPH produced [49]. To circumvent this, we performed CMA in order to identify the targets for cofactor specificity engineering to improve the NADPH pool which can enhance DDDA yield. From the CMA results, it was observed that the DDDA yield can be improved by increasing NADPH regeneration through modification of cofactor specificity from NAD to NADP. Among the targets found, changing the cofactor specificity of GAPD and MDH from NAD to NADP was found to give the best improvement in DDDA yield (Table 2).

To achieve a high-level production of DCA in yeast, we propose to enhance the supply of NADPH to the $\omega$ oxidation pathway. Hence, a multi-step metabolic engineering strategy can be devised to simultaneously modify the glycolysis step, optimize the NADPH supply and to enhance the activity of some NADPH producing enzymes.

\section{Effect of branched-chain amino acids supplementation on DCA production}

In most recombinant DCAs production studies, the hydrophobic substrates such as alkane and fatty acid methyl ester (FAME) were used for the biotransformation [5]. As such, glucose is used as the carbon source for the growth before inducing the $\omega$-oxidation pathway using alkane. However, it has been shown in Y. lipolytica that lipid accumulation can occur de novo without exogenous supply of hydrophobic substrate, laying the grounds for a possibility to be used for de novo DCAs production as well, from primary carbon sources, i.e., glucose or glycerol [50]. In addition to this, the ability to efficiently produce acetyl-CoA by metabolizing other carbon sources in the medium will drive the important precursor towards DCAs production. Presently, there are evidences implicating a strong correlation between lipid accumulation and leucine metabolism in S. cerevisiae [51] and $Y$. lipolytica [52]. Taking the clue from lipid accumulation studies on $Y$. lipolytica, we sought to explore the effect of BCAA supplementation on acetylCoA pool, which can be produced via degradation pathway of BCAA. In order to theoretically analyze the flux distribution and flux-sum changes that occur when BCAA is supplemented, we maximized the DDDA production using glucose as the primary carbon source supplemented with $10 \mathrm{C}-\mathrm{mmol} / \mathrm{gDCW}-\mathrm{hr}$ of leucine, isoleucine, or valine. To elucidate the difference in pathway utilization and metabolite turn-over in different amino acids during DDDA production, we prepared the heat map of flux distribution and flux-sum as shown in Fig. 5. It can be seen that acetyl-CoA being critical determinant of DCAs synthesis was produced at the highest level on valine supplementation followed by leucine which correlated with DDDA turn over trend. Apart from the role of BCAAs in acetyl-CoA production, supplementing amino acids decreases the primary carbon demand in amino acid biosynthesis for biomass formation. This extra carbon can then be diverted to DCAs biosynthesis. This observation is in close resemblance with nitrogen starvation condition for lipid accumulation because in nitrogen limiting condition, biosynthesis of amino acid seizes and carbon present then can be utilized in other biosynthetic pathways. From our simulation, we have identified that glucose supplemented with valine or leucine can increase the growth as well as de novo accumulation of DCAs in $Y$. lipolytica. This is the first report implicating a prominent role of valine degradation as an alternate route of acetylCoA biosynthesis in Y. lipolytica, experimental validation of which can give insight to intricate correlation between fatty acid biosynthesis and amino acid degradation.

\section{Conclusion}

The GSMM of $Y$. lipolytica, iYLI647, was reconstructed using $i$ MK735 as a scaffold and the relevant information from other available $Y$. lipolytica models. iYLI647 consists of 1347 reactions and 647 genes. The biomass

Table 2 Cofactor specificity engineering targets by CMA to increase DCAs production

\begin{tabular}{llll}
\hline Reaction & Reaction Definition & \multicolumn{2}{l}{ Yield of DDDA (mmol/gDCW-hr) } \\
\cline { 3 - 4 } & & NAD & NADP \\
\hline MDH & mal_L[c] + nad $[c]<=>h[c]+$ nadh $[c]+$ oaa $[c]$ & 2.601 & 2.781 \\
GAPD & g3p $[c]+$ nad $[c]+$ pi $[c]<=>13 d p g[c]+h[c]+$ nadh $[c]$ & 2.601 & 2.778 \\
\hline
\end{tabular}




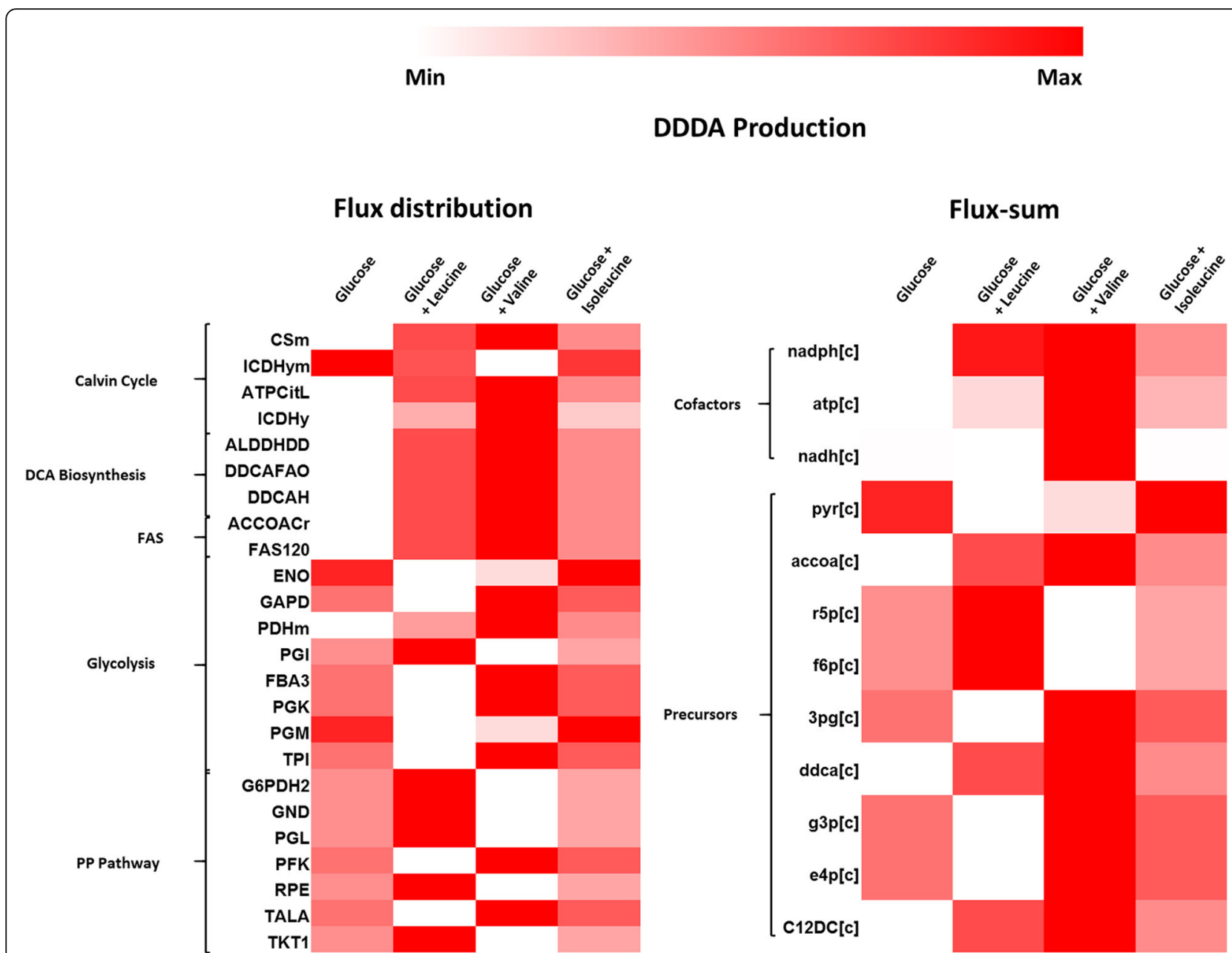

Fig. 5 Flux distribution and flux-sum. Heat map showing flux distribution and flux-sum of important reactions and metabolites, respectively, during DDDA production

equations were carefully formulated with various experimental information of $Y$. lipolytica, which is perhaps the reason for accurate model prediction. The potential of de novo DCAs production in $Y$. lipolytica combined with a model-driven strain design for metabolic engineering and media optimization strategies were then evaluated using the reconstructed $i$ YLI647. The flux towards DDDA production was increased following the overexpression and deletion of few reactions and the model-based strain design gives us a good starting point to explore the metabolic capabilities of $Y$. lipolytica to produce fatty acid derived products. Moreover, the workflow and procedure presented in this analysis can be utilized as a platform to perform similar analyses with different organisms.

\section{Methods}

\section{Metabolic network reconstruction}

We chose all four separately developed publicly available metabolic network reconstructions, iNL895, iYL619, $i \mathrm{MK} 735$, and $i$ Yali4, to compare and select the best suited as scaffold model. Following our qualitative and quantitative comparison, we chose $i$ MK735 as a scaffold model and proceeded to manual curation to expand the model coverage and characteristics. To do this, using literature data, we verified the presence of reactions and relevance in $Y$. lipolytica metabolism. Then, we added $\omega$-oxidation pathway to convert fatty acids to DCAs, and also the subsequent degrading $\beta$-oxidation pathway. Following the literature evidence and its established importance in fatty acid metabolism, we also included BCAA degradation pathways. Furthermore, we checked the mass balance of reactions and made appropriate changes to make stoichiometrically balanced reactions. In addition, we derived the biomass equation in carbon and nitrogen limited conditions using updated and relevant literature sources. Using refined biomass equation, we simulated the model to identify the loops, and missing link reactions using GapFinder [53]. Loops were removed by changing the directionality of coupled 
reaction, or removing the non-metabolic lumped reactions, whereas, gaps were filled with reactions from orthologs or introducing transport/sink reaction.

\section{Constraints-based flux analysis}

The cellular metabolism of $Y$. lipolytica was simulated under varying environmental conditions using constraintsbased flux analysis. The biomass reaction was maximized to simulate the growth under various culture conditions as described elsewhere [54-56]. The maximization of biomass is subjected to stoichiometric and capacity constraints, which can mathematically be formulated as:

$$
\max Z_{1}=\sum_{j} c_{j} v_{j}
$$

$$
\begin{aligned}
& \text { s.t. } \quad \sum_{j} S_{i j} v_{j}=0 \quad \forall \text { metabolite } i \\
& v_{j}^{\min } \leq v_{j} \leq v_{j}^{\max } \quad \forall \text { reaction } j
\end{aligned}
$$

where $S_{i j}$ refers to the stoichiometric coefficient of metabolite $i$ involved in reaction $j, v_{j}$ denotes to the flux or specific rate of metabolic reaction $j, v_{j}^{\min }$ and $v_{j}^{\max }$ represent the lower and upper limits on the flux of reaction $j$, respectively; and $Z_{1}$ corresponds to the cellular objective as a linear function of all the metabolic reactions where the relative weights are determined by the coefficient $c_{j}$. In this study, the constraints-based flux analysis problems were solved using COBRA toolbox [57].

\section{In silico model-based strain design}

Four in silico strain design approaches have been employed to identify metabolic engineering targets for overproducing DCA from glucose.

\section{Genetic design by local search (GDLS)}

GDLS strain design algorithm [33] was implemented in COBRA toolbox in Matlab, searching up to maximum of 5 knockout reactions with the outer objective of maximizing DDDA exchange flux.

\section{Flux activity analysis}

To identify the upregulation and downregulation gene/ reaction targets which lead to the enhanced production of desired compound, we first needed to quantify the flux activity of all reactions in the wild-type strain. Flux activity, $f_{j}$, is defined as the absolute value of reaction flux, $v_{j}$ [34]. This can be determined by first solving the constraints-based flux analysis problem (Eq. 1) with biomass maximization as objective, and then obtaining the absolute values of individual reaction fluxes. Next, we solved the below-mentioned mixed-integer linear programming (MILP) problem to identify the maximum and minimum flux activities to determine the feasible ranges of individual reactions such that they can be upregulated and downregulated within this limit:

$$
\max / \min f_{j}
$$

Subject to:

$$
\begin{aligned}
& \sum_{j} S_{i j} v_{j}=0 \\
& \alpha_{j} \leq v_{j} \leq \beta_{j} \\
& v_{j}=f_{j}^{+}-f_{j}^{-} \\
& f_{j}^{+} \geq 0 \quad ; \quad f_{j}^{-} \geq 0 \\
& f_{j}^{+} \leq I_{j}^{+} \cdot M \quad ; \quad f_{j}^{-} \leq I_{j}^{-} \cdot M \\
& I_{j}^{+} \in\{0,1\} \quad ; \quad I_{j}^{-} \in\{0,1\} \\
& I_{j}^{+}+I_{j}^{-}=1
\end{aligned}
$$

Where, $\alpha_{j}$ and $\beta_{j}$ are upper and lower bounds of fluxes, respectively. The $f_{j}^{+}$and $f_{j}^{*}$ are the positive two variables into which the flux, $v_{j}$, is decomposed. It is observed that $f_{j}^{+}+f_{j}^{-}=\left|f_{j}^{+}-f_{j}^{-}\right|$, if and only if either $f_{j}^{+}$or $f_{j}^{-}$is equal to zero. This condition was introduced by new binary variables $I_{j}^{+}$and $I_{j}^{-}$which are when multiplied with a large integer which should be larger than the flux of experimentally measured value, $\mathrm{M}$, will be always lesser than $f_{j}^{+}$and $f_{j}^{-}$, respectively. Additionally, the constraint $I_{j}^{+}+I_{j}^{-}=1$ is also introduced to ensure that either $f_{j}^{+}$or $f_{j}^{-}$are equal to zero.

Once the reference flux activities are established, i.e. wild-type, maximum and minimum values, we then solve the below mentioned MILP problem to analyze the effects of perturbing a particular flux activity on cellular growth.

$$
\max v_{\text {biomass }}
$$

Subject to:

$$
\begin{aligned}
& \sum_{j} S_{i j} v_{j}=0 \\
& \alpha_{j} \leq v_{j} \leq \beta_{j} \\
& v_{j}=f_{j}^{+}-f_{j}^{-} \\
& f_{j}^{+} \geq 0 \quad ; \quad f_{j}^{-} \geq 0 \\
& f_{j}^{+} \leq I_{j}^{+} \cdot M \quad ; \quad f_{j}^{-} \leq I_{j}^{-} \cdot M \\
& I_{j}^{+} \in\{0,1\} \quad ; \quad I_{j}^{-} \in\{0,1\} \\
& I_{j}^{+}+I_{j}^{-}=1 \\
& (\mathrm{C} 1): f_{j}^{+}+f_{j}^{-} \leq f_{j}^{\min }+k_{\mathrm{att}}\left(f_{j}^{W T}-f_{j}^{\min }\right)
\end{aligned}
$$


OR

$$
(\mathrm{C} 2): f_{j}^{+}+f_{j}^{-} \geq f_{j}^{W T}+k_{\mathrm{int}}\left(f_{j}^{\max }-f_{j}^{W T}\right)
$$

Where, constraints $(\mathrm{C} 1)$ and $(\mathrm{C} 2)$ are applicable for upregulation and downregulation problems, respectively. Parameters $k_{\text {att }}$ and $k_{\text {int }}$ are gradually varied between 0 and 1 in steps of 0.1 to analyze the effect of reaction upregulation between minimal and wild-type values, and reaction downregulation between the wild-type and maximal values, respectively.

Finally, the objective value obtained from the solution of (Eq. 3) is used as the lower limit for cell growth in the fourth step whereby (Eq. 3) is solved again with the targeted product as the objective function. The corresponding mathematical formulation is as follows:

$\min v_{E X \_s u c c}$

Subject to:

$$
\begin{aligned}
& v_{\text {biomass }} \geq B_{j, k} \\
& \sum_{j} S_{i j} v_{j}=0 \\
& \alpha_{j} \leq v_{j} \leq \beta_{j} \\
& v_{j}=f_{j}^{+}-f_{j}^{-} \\
& f_{j}^{+} \geq 0 \quad ; \quad f_{j}^{-} \geq 0 \\
& f_{j}^{+} \leq I_{j}^{+} \cdot M \quad ; \quad f_{j}^{-} \leq I_{j}^{-} \cdot M \\
& I_{j}^{+} \in\{0,1\} \quad ; \quad I_{j}^{-} \in\{0,1\} \\
& I_{j}^{+}+I_{j}^{-}=1 \quad \\
& (\mathrm{C} 1): f_{j}^{+}+f_{j}^{-} \leq f_{j}^{\min }+k_{\mathrm{att}}\left(f_{j}^{W T}-f_{j}^{\min }\right)
\end{aligned}
$$

OR

$$
(\mathrm{C} 2): f_{j}^{+}+f_{j}^{-} \geq f_{j}^{W T}+k_{\mathrm{int}}\left(f_{j}^{\max }-f_{j}^{W T}\right)
$$

where $B_{j, k}$ is the maximum biomass obtainable while solving problem (Eq. 3) for $j^{\text {th }}$ reaction at $k^{\text {th }}$ upregulation/downregulation levels. All the optimization problems were solved using the GAMS IDE software version 22.4 with IBM ILOG CPLEX solver.

\section{Transcriptomics-based strain optimization tool (tSOT)}

Since optimality assumption based FBA algorithms ignores the regulatory consideration while strain designing, we implemented transcriptomics-based strain optimization tool (tSOT) [35] to identify metabolic engineering targets based on transcriptomic data integrated in the model. A comprehensive time-course transcriptomic profile from the culture of $Y$. lipolytica, during a controlled fed-batch on glucose, was used as transcriptomic data obtained from Gene Expression Omnibus (GEO) database using accession number GSE29046 [40].

\section{Cofactor modification analysis (CMA)}

Since the $\omega$-oxidation pathway is an oxidative process and requires cofactor optimization to maximize theoretical yield, we used CMA to identify the cofactor specificity engineering target which can increase the yield of DDDA. CMA was implemented as described in our previous work [36]. Mathematically, the bi-level mixed-integer nonlinear programming (MINLP) optimization problem specific to the CMA can be represented as follows:

$$
\begin{gathered}
\max \phi_{\text {product }}=0.5 \sum_{j}\left|S_{\text {product }, j} v_{j}\right| \\
\text { s.t. }\left[\begin{array}{cc}
\max & v_{\text {biomass }} \\
\text { s.t. } & \sum_{j}\left(S_{i j} v_{j}+S_{i j}^{c \text { Mod }} v_{j}^{c \text { Mod }}\right)=0 \forall \text { metabolite } i \\
& v_{\text {biomass }} \geq v_{\text {biomass }}^{\min } \\
\left(1-y_{j}^{c \text { Mod }}\right) \cdot v_{j}^{\min } \leq v_{j} \leq\left(1-y_{j}^{c \text { Mod }}\right) \cdot v_{j}^{\max } \\
y_{j}^{c \text { Mod d }} \cdot v_{j}^{\min } \leq v_{j}^{c \text { Mod }} \leq y_{j}^{c \text { Mod }} \cdot v_{j}^{\max } \\
y_{j}^{c \text { Mod }}=\{0,1\} \forall \text { reaction } j
\end{array}\right] \\
\sum_{j} y_{j}^{c \text { Mod }} \leq k
\end{gathered}
$$

Where, $S_{i j}^{c M o d}$ is the cofactor modified stoichiometric matrix where the coefficients are same as $S_{i j}$, except the reactions which involve either $\mathrm{NAD}(\mathrm{H})$ or $\mathrm{NADP}(\mathrm{H})$. These reactions are swapped for cofactors in the $S_{i j}^{c M o d}$ matrix such that $S_{N A D(H), j}=S_{N A D P(H), j}^{c M o d}$ and $S_{N A D P(H), j}=S_{N A D(H), j}^{c M o d}$ . $v_{j}^{c M o d}$ is the flux through the cofactor modified reaction and $v_{\text {biomass }}^{\min }$ is the minimum amount of biomass that needs to be produced. The binary variable $y_{j}^{c M o d}$ ensures that the cofactor associated reactions are allowed to carry flux either with its original or swapped cofactor but not both. The number of cofactor switches allowed in a particular simulation is controlled by the number, $k$ and which is fixed at 1 for all simulations in this work. The bi-level MINLP problem was reformulated as a single-level MINLP problem using the primal dual transformation as implemented earlier [58]. The MILP optimization problem was solved using the GAMS IDE software version 22.4 with IBM ILOG CPLEX solver.

\section{Flux-sum}

In the constraints-based flux analysis, there is no accumulation of intermediate metabolites due to the steadystate condition. However, the turnover rate, which is also equivalent to the total consumption or production rate, of the intermediates can be nonzero, which is defined as 
their flux-sum [59]. Since the overall consumption and generation rates are equal under the steady-state assumption, the flux-sum of metabolite $i$ can be formulated as $\Phi_{i}=0.5 \sum\left|S_{i j} v_{j}\right|$. Each term in this summation series gives us the absolute rate of consumption/generation of metabolite $i$ due to reaction $j$ and thus by halving the sum of these terms, we can obtain the overall turnover rate for metabolite $i$.

\section{Additional files}

Additional file 1: Changed, added and deleted reactions in iYLI647 model in comparison with iMK735 scaffold model. (XLSX 22 kb)

Additional file 2: SMBL file of Y YLI647. (XML $2312 \mathrm{~kb}$ )

Additional file 3: Biomass composition of $Y$. lipolytica in C- and Nlimited conditions and GAM and NGAM calculations. (DOCX 52 kb)

\section{Acknowledgements}

We thank Dr. Jung-Oh Ahn (Korea Research Institute of Bioscience and Biotechnology) for help and discussion.

\section{Funding}

This work was supported by the Academic Research Fund (R-279-000-476112) of the National University of Singapore, Biomedical Research Council of A*STAR (Agency for Science, Technology and Research), Singapore, the Global R\&D project program (N011500017), Ministry of Trade, Industry and Energy (MOTIE), Republic of Korea and the Next-Generation BioGreen 21 Program of the Rural Development Administration, Republic of Korea (Systems and Synthetic Agrobiotech Center; grant no. PJ01334605). Publication cost was covered by NUS SynCTI program.

\section{Availability of data and materials}

All data generated or analyzed during this study are included in this published article and its supplementary information files.

\section{About this supplement}

This article has been published as part of BMC Systems Biology Volume 12 Supplement 2, 2018: Proceedings of the 28th International Conference on Genome Informatics: systems biology. The full contents of the supplement are available online at https://bmcsystbiol.biomedcentral.com/articles/ supplements/volume-12-supplement-2.

\section{Authors' contributions}

PM, NRL and DYL conceived and designed the in silico study. PM, NRL, ML and MSK performed the computational simulations and drafted the manuscript. PM, NRL, BGK and DYL revised the manuscript. All authors read and approved the final manuscript.

\section{Ethics approval and consent to participate}

Not applicable.

\section{Consent for publication}

Not applicable.

\section{Competing interests}

The authors declare that they have no competing interests.

\section{Publisher's Note}

Springer Nature remains neutral with regard to jurisdictional claims in published maps and institutional affiliations.

\section{Author details}

${ }^{1}$ NUS Synthetic Biology for Clinical and Technological Innovation (SynCTI), Life Sciences Institute, National University of Singapore, 28 Medical Drive, Singapore 117456, Singapore. ${ }^{2}$ Bioprocessing Technology Institute, Agency for Science, Technology and Research (A*STAR), 20 Biopolis Way, \#06-01,
Centros, Singapore 138668, Singapore. ${ }^{3}$ School of Chemical and Biological Engineering, Institute of Molecular Biology and Genetics, and Bioengineering Institute, Seoul National University, 1 Gwanak-ro, Gwanak-gu, Seoul 151-742, Republic of Korea. ${ }^{4}$ School of Chemical Engineering, Sungkyunkwan University, 2066 Seobu-ro, Jangan-gu, Suwon, Gyeonggi-do 16419, Republic of Korea.

Published: 19 March 2018

\section{References}

1. Cornils B, Lappe P. Dicarboxylic acids, Aliphatic. Ullmann's Encycl Ind Chem. 2012;11:287-304. https://doi.org/10.1002/14356007.a08.

2. Polen T, Spelberg M, Bott M. Toward biotechnological production of adipic acid and precursors from biorenewables. J Biotechnol. 2013:75-84. https:// doi.org/10.1016/j.jbiotec.2012.07.008.

3. Green KD, Turner MK, Woodley JM. Candida Cloacae oxidation of long-chain fatty acids to dioic acids. Enzym Microb Technol. 2000;27:205-11. https:// doi.org/10.1016/S0141-0229(00)00217-9.

4. Jiao P, Ma S, Hua Y, Huang Y, Cao Z. Isolation and enzyme determination of Candida Tropicalis mutants for DCA production. J Gen Appl Microbiol. 2000; 46:245-9. https://doi.org/10.2323/jgam.46.245

5. Smit MS, Mokgoro MM, Setati E, Nicaud J-M. Alpha,omega-dicarboxylic acid accumulation by acyl-CoA oxidase deficient mutants of Yarrowia lipolytica. Biotechnol Lett. 2005;27:859-64. https://doi.org/10.1007/s10529-005-6719-1.

6. Chan EC, Cheng CS, Hsu YH. Continuous production of dicarboxylic acid by immobilized Pseudomonas Aeruginosa cells. J Ferment Bioeng. 1997:83: 157-60. https://doi.org/10.1016/S0922-338X(97)83575-1.

7. Chan EC, Kuo J. Biotransformation of dicarboxylic acid by immobilized Cryptococcus cells. Enzym Microb Technol. 1997;20:585-9. https://doi.org/ 10.1016/S0141-0229(96)00198-6.

8. Sathesh-Prabu C, Lee SK. Production of long-chain alpha,omega-dicarboxylic acids by engineered Escherichia Coli from renewable fatty acids and plant oils. J Agric Food Chem. 2015;63:8199-208. https://doi.org/10.1021/acs.jafc.5b03833.

9. Ledesma-Amaro R, Nicaud JM. Yarrowia lipolytica as a biotechnological chassis to produce usual and unusual fatty acids. Prog Lipid Res. 2016;61: 40-50. https://doi.org/10.1016/j.plipres.2015.12.001.

10. Eschenfeldt WH, Zhang $Y$, Samaha H, Stols L, Eirich LD, Wilson $C R$, et al. Transformation of fatty acids catalyzed by cytochrome P450 monooxygenase enzymes of Candida Tropicalis. Appl Environ Microbiol. 2003;69:5992-9. https://doi.org/10.1128/AEM.69.10.5992-5999.2003.

11. Gatter M, Förster A, Bär K, Winter M, Otto C, Petzsch P, et al. A newly identified fatty alcohol oxidase gene is mainly responsible for the oxidation of long-chain $\omega$-hydroxy fatty acids in Yarrowia lipolytica. FEMS Yeast Res. 2014;14:858-72. https://doi.org/10.1111/1567-1364.12176.

12. Eirich LD, Craft DL, Steinberg L, Asif A, Eschenfeldt WU, Stols L, et al. Cloning and characterization of three fatty alcohol oxidase genes from Candida Tropicalis strain ATCC 20336. Appl Environ Microbiol. 2004;70:48729. https://doi.org/10.1128/AEM.70.8.4872-4879.2004.

13. Iwama R, Kobayashi S, Ishimaru C, Ohta A, Horiuchi H, Fukuda R. Functional roles and substrate specificities of twelve cytochromes $\mathrm{P} 450$ belonging to CYP52 family in n-alkane assimilating yeast Yarrowia lipolytica. Fungal Genet Biol. 2016;91:43-54. https://doi.org/10.1016/j.fgb.2016.03.007.

14. Broadway NM, Dickinson FM, Ratledge C. The enzymology of dicarboxylic-acid formation by Corynebacterium Sp strain 7E1C grown on N-alkanes. J Gen Microbiol. 1993;139:1337-44. https://doi.org/10.1099/00221287-139-6-1337.

15. Nicaud J, Thevenieau F, Le Dall M, Marchal R. Production of dicarboxylic acids by improved mutant strains of yarrowia lipolytica. US pat app 11/; 2005. p. 1. Available: http://www.google.com/patents/US20100041115

16. Simeonidis E, Price ND. Genome-scale modeling for metabolic engineering. J Ind Microbiol Biotechnol. 2015:327-38. https://doi.org/10.1007/s102950e14-1576-3.

17. Alper H, Jin YS, Moxley JF, Stephanopoulos G. Identifying gene targets for the metabolic engineering of lycopene biosynthesis in Escherichia Coli. Metab Eng. 2005;7:155-64. https://doi.org/10.1016/j.ymben.2004.12.003.

18. Alper H, Miyaoku K, Stephanopoulos G. Construction of lycopeneoverproducing E. Coli strains by combining systematic and combinatorial gene knockout targets. Nat Biotechnol. 2005;23:612-6. https://doi.org/10. 1038/nbt1083.

19. Bro C, Regenberg B, Förster J, Nielsen J. In silico aided metabolic engineering of Saccharomyces Cerevisiae for improved bioethanol production. Metab Eng. 2006:8:102-11. https://doi.org/10.1016/j.ymben.2005.09.007. 
20. Sun Z, Meng H, Li J, Wang J, Li Q, Wang Y, et al. Identification of novel knockout targets for improving terpenoids biosynthesis in Saccharomyces Cerevisiae. PLoS One. 2014;9 https://doi.org/10.1371/journal.pone.0112615.

21. Loira N, Dulermo T, Nicaud J-M, Sherman DJ. A genome-scale metabolic model of the lipid-accumulating yeast Yarrowia lipolytica. BMC Syst Biol. BioMed Central Ltd. 2012;6:35. https://doi.org/10.1186/1752-0509-6-35.

22. Pan $\mathrm{P}$, Hua $\mathrm{Q}$. Reconstruction and in silico analysis of metabolic network for an oleaginous yeast, Yarrowia lipolytica. PLoS One. 2012;7:e51535. https:// doi.org/10.1371/journal.pone.0051535.

23. Kavšček M, Bhutada G, Madl T, Natter K, Krainer F, Dietzsch C, et al. Optimization of lipid production with a genome-scale model of Yarrowia lipolytica. BMC Syst Biol. 2015;9:72. https://doi.org/10.1186/s12918-015-0217-4

24. Kerkhoven EJ, Pomraning KR, Baker SE, Nielsen J. Regulation of amino-acid metabolism controls flux to lipid accumulation in Yarrowia lipolytica. Npj. Syst Biol Appl. 2016;2:16005. https://doi.org/10.1038/npjsba.2016.5.

25. Duarte NC, Herrgård MJ, Palsson B $\varnothing$. Reconstruction and validation of Saccharomyces Cerevisiae iND750, a fully compartmentalized genome-scale metabolic model. Genome Res. 2004;14:1298-309. https://doi.org/10.1101/ gr.2250904.

26. Blazeck J, Liu L, Knight R, Alper HS. Heterologous production of pentane in the oleaginous yeast Yarrowia lipolytica. J Biotechnol. 2013;165:184-94. https://doi.org/10.1016/j.jbiotec.2013.04.003.

27. Vorapreeda T, Thammarongtham C, Cheevadhanarak S, Laoteng K. Alternative routes of acetyl-CoA synthesis identified by comparative genomic analysis: involvement in the lipid production of oleaginous yeast and fungi. Microbiology. 2012;158:217-28. https://doi.org/10.1099/mic.0.051946-0.

28. Van Maris AJA, Luttik MAH, Winkler AA, Van Dijken JP, Pronk JT. Overproduction of threonine aldolase circumvents the biosynthetic role of pyruvate decarboxylase in glucose-limited chemostat cultures of Saccharomyces Cerevisiae. Appl Environ Microbiol. 2003;69:2094-9. https:// doi.org/10.1128/AEM.69.4.2094-2099.2003.

29. Papanikolaou S, Aggelis G. Lipid production by Yarrowia lipolytica growing on industrial glycerol in a single stage continuous culture. Bioresour Technol. 2002;82:43-9. https://doi.org/10.1186/s13068-015-0286-z.

30. Workman M, Holt P, Thykaer J. Comparing cellular performance of Yarrowia lipolytica during growth on glucose and glycerol in submerged cultivations. AMB Express. 2013;3:58. https://doi.org/10.1186/2191-0855-3-58.

31. Dulermo T, Lazar Z, Dulermo R, Rakicka M, Haddouche R, Nicaud JM. Analysis of ATP-citrate lyase and malic enzyme mutants of Yarrowia lipolytica points out the importance of mannitol metabolism in fatty acid synthesis. Biochim Biophys Acta - Mol Cell Biol Lipids. Elsevier B.V. 2015; 1851:1107-17. https://doi.org/10.1016/j.bbalip.2015.04.007.

32. Mostafa N a, Maher A, Abdelmoez W. Production of mono-, di-, and triglycerides from waste fatty acids through esterification with glycerol. Adv Biosci Biotechnol. 2013;4:900-7. http://dx.doi.org/10.4236/abb.2013.49118

33. Lun DS, Rockwell G, Guido NJ, Baym M, Kelner JA, Berger B, et al. Largescale identification of genetic design strategies using local search. Mol Syst Biol. 2009;5:296. https://doi.org/10.1038/msb.2009.57.

34. Chung BK, Selvarasu S, Andrea C, Ryu J, Lee H, Ahn J, et al. Genome-scale metabolic reconstruction and in silico analysis of methylotrophic yeast Pichia Pastoris for strain improvement. Microb Cell Factories. 2010;9:50. https://doi.org/10.1186/1475-2859-9-50.

35. Kim M, Yi JS, Lakshmanan M, Lee DY, Kim BG. Transcriptomics-based strain optimization tool for designing secondary metabolite overproducing strains of Streptomyces Coelicolor. Biotechnol Bioeng. 2016;113:651-60. https://doi. org/10.1002/bit.25830.

36. Lakshmanan M, Chung BK-S, Liu C, Kim S-W, Lee D-Y. Cofactor modification analysis: a computational framework to identify cofactor specificity engineering targets for strain improvement. J Bioinforma Comput Biol. 2013; 11:1343006. https://doi.org/10.1142/S0219720013430063.

37. Zaslaver A, Mayo AE, Rosenberg R, Bashkin P, Sberro H, Tsalyuk M, et al. Just-in-time transcription program in metabolic pathways. Nat Genet. 2004; 36:486-91. https://doi.org/10.1038/ng1348

38. Little JW, Shepley DP, Wert DW. Robustness of a gene regulatory circuit. EMBO J. 1999;18:4299-307. https://doi.org/10.1093/emboj/18.15.4299.

39. Iwama R, Kobayashi S, Ohta A, Horiuchi H, Fukuda R. Alcohol dehydrogenases and an alcohol oxidase involved in the assimilation of exogenous fatty alcohols in Yarrowia lipolytica. FEMS Yeast Res. 2015;15:112. https://doi.org/10.1093/femsyr/fov014.

40. Morin N, Cescut J, Beopoulos A, Lelandais G, Le Berre V, Uribelarrea J-L, et al. Transcriptomic analyses during the transition from biomass production to lipid accumulation in the oleaginous yeast Yarrowia lipolytica. PLoS One. 2011:6:e27966. https://doi.org/10.1371/journal.pone.0027966.

41. Ratledge $\mathrm{C}$. The role of malic enzyme as the provider of NADPH in oleaginous microorganisms: a reappraisal and unsolved problems. Biotechnol Lett. 2014;36:1557-68. https://doi.org/10.1007/s10529-014-1532-3.

42. Miflin BJ, Habash DZ. The role of glutamine synthetase and glutamate dehydrogenase in nitrogen assimilation and possibilities for improvement in the nitrogen utilization of crops. J Exp Bot. 2002;53:979-87. https://doi.org/ 10.1093/jexbot/53.370.979.

43. Qiao K, Imam Abidi SH, Liu H, Zhang H, Chakraborty S, Watson N, et al. Engineering lipid overproduction in the oleaginous yeast Yarrowia lipolytica. Metab Eng. 2015;29:56-65. https://doi.org/10.1016/j.ymben.2015.02.005.

44. Gao C, Zhang L, Xie Y, Hu C, Zhang Y, Li L, et al. Production of (3S)-acetoin from diacetyl by using stereoselective NADPH-dependent carbonyl reductase and glucose dehydrogenase. Bioresour Technol. 2013;137:111-5. https://doi.org/10.1016/j.biortech.2013.02.115.

45. Sun JA, Zhang LY, Rao B, Shen YL, Wei DZ. Enhanced acetoin production by Serratia Marcescens H32 with expression of a water-forming NADH oxidase. Bioresour Technol. 2012;119:94-8. https://doi.org/10.1016/j.biortech.2012.05.108.

46. Takeno S, Murata R, Kobayashi R, Mitsuhashi S, Ikeda M. Engineering of Corynebacterium glutamicum with an NADPH-generating glycolytic pathway for L-lysine production. Appl Environ Microbiol. 2010;76:7154-60. https://doi.org/10.1128/AEM.01464-10

47. Verho $R$, Londesborough J, Penttilä $M$, Richard $P$. Engineering redox cofactor regeneration for improved pentose fermentation in Saccharomyces Cerevisiae. Appl Environ Microbiol. 2003;69:5892-7. https://doi.org/10.1128/ AEM.69.10.5892-5897.2003.

48. Shi A, Zhu X, Lu J, Zhang X, Ma Y. Activating transhydrogenase and NAD kinase in combination for improving isobutanol production. Metab Eng. 2013;16:1-10. https://doi.org/10.1016/j.ymben.2012.11.008.

49. Wasylenko TM, Ahn WS, Stephanopoulos G. The oxidative pentose phosphate pathway is the primary source of NADPH for lipid overproduction from glucose in Yarrowia lipolytica. Metab Eng. 2015;30:2739. https://doi.org/10.1016/j.ymben.2015.02.007.

50. Seip J, Jackson R, He H, Zhu Q, Hong SP. Snf1 is a regulator of lipid accumulation in Yarrowia lipolytica. Appl Environ Microbiol. 2013;79:736070. https://doi.org/10.1128/AEM.02079-13.

51. Kamisaka Y, Tomita N, Kimura K, Kainou K, Uemura H. DGA1 (diacylglycerol acyltransferase gene) overexpression and leucine biosynthesis significantly increase lipid accumulation in the $\Delta$ snf2 disruptant of Saccharomyces Cerevisiae. Biochem J. 2007;408:61. https://doi.org/10.1042/bj20070449.

52. Blazeck J, Hill A, Liu L, Knight R, Miller J, Pan A, et al. Harnessing Yarrowia lipolytica lipogenesis to create a platform for lipid and biofuel production. Nat Commun. Nature Publishing Group. 2014;5:3131. https://doi.org/10. 1038/ncomms4131.

53. Satish Kumar V, Dasika MS, Maranas CD. Optimization based automated curation of metabolic reconstructions. BMC Bioinformatics. 2007:8:212. https://doi.org/10.1186/1471-2105-8-212.

54. Orth JD, Thiele I, Palsson B $\varnothing$. What is flux balance analysis? Nat Biotechnol. 2010;28:245-8. https://doi.org/10.1038/nbt.1614.

55. Oberhardt MA, Palsson BØ, Papin JA. Applications of genome-scale metabolic reconstructions. Mol Syst Biol. 2009;5:320. https://doi.org/10.1038/ msb.2009.77.

56. Lee JM, Gianchandani EP, Papin JA. Flux balance analysis in the era of metabolomics. Brief Bioinform. 2006:140-50. https:/doi.org/10.1093/bib/bbl007.

57. Schellenberger J, Que R, Fleming RMT, Thiele I, Orth JD, Feist AM, et al. Quantitative prediction of cellular metabolism with constraint-based models: the COBRA toolbox v2.0. Nat Protoc. 2011;6:1290-307. https://doi. org/10.1038/nprot.2011.308.

58. Lakshmanan M, Yu K, Koduru L, Lee D-Y. In silico model-driven cofactor engineering strategies for improving the overall NADP(H) turnover in microbial cell factories: J Ind Microbiol Biotechnol. Springer Berlin Heidelberg; 2015. https://doi.org/10.1007/s10295-015-1663-0.

59. Kim P-J, Lee D-Y, Kim TY, Lee KH, Jeong H, Lee SY, et al. Metabolite essentiality elucidates robustness of Escherichia Coli metabolism. Proc Natl Acad Sci U S A. 2007;104:13638-42. https://doi.org/10.1073/pnas. 0703262104. 